\title{
UV-visible-near infrared photoabsorption and photodetection using close-packed metallic gold nanoparticle network
}

Cite as: J. Appl. Phys. 107, 053510 (2010); https://doi.org/10.1063/1.3310367

Submitted: 09 September 2009 . Accepted: 14 January 2010 . Published Online: 04 March 2010

Xian Ning Xie, Yu Lin Zhong, Mohan S. Dhoni, Yilin Xie, Kian Ping Loh, Chorng Haur Sow, Wei Ji, and Andrew Thye Shen Wee

\section{ARTICLES YOU MAY BE INTERESTED IN}

Evidence of an enhanced interband absorption in Au nanoparticles: Size-dependent electronic structure and optical properties

Applied Physics Letters 87, 143105 (2005); https://doi.org/10.1063/1.2077834

Enhanced semiconductor optical absorption via surface plasmon excitation in metal nanoparticles

Applied Physics Letters 86, 063106 (2005); https://doi.org/10.1063/1.1855423

Surface plasmon-enhanced photodetection in $\mathrm{MoTe}_{2}$ phototransistors with Au nanoparticles Applied Physics Letters 115, 142102 (2019); https://doi.org/10.1063/1.5116644

Challenge us. What are your needs for periodic signal detection?

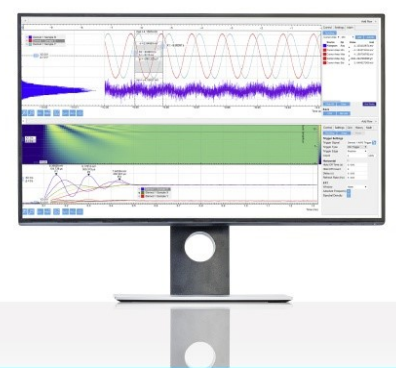

- Zurich Instruments 


\title{
UV-visible-near infrared photoabsorption and photodetection using close-packed metallic gold nanoparticle network
}

\author{
Xian Ning Xie, ${ }^{1, a)}$ Yu Lin Zhong, ${ }^{2}$ Mohan S. Dhoni, ${ }^{3}$ Yilin Xie, ${ }^{3}$ Kian Ping Loh, ${ }^{1,2}$ \\ Chorng Haur Sow, ${ }^{3}$ Wei Ji, ${ }^{3}$ and Andrew Thye Shen Wee ${ }^{1,3}$ \\ ${ }^{1}$ NUS Nanoscience and Nanotechnology Initiative (NUSNNI), National University of Singapore, Singapore \\ 117542 \\ ${ }^{2}$ Department of Chemistry, National University of Singapore, Singapore 117543 \\ ${ }^{3}$ Department of Physics, National University of Singapore, 2 Science Drive 3, Singapore 117542
}

(Received 9 September 2009; accepted 14 January 2010; published online 4 March 2010)

\begin{abstract}
Photocurrent generation and photodetection are usually based on semiconductor crystals including $\mathrm{Si}, \mathrm{CdS}$, and $\mathrm{PbS}$. This work reports the enhanced photoabsorption and photodetection of close-packed metallic Au nanoparticles (NPs) in the UV-VIS (visible)-NIR (near infrared) region. Photoabsorption in the UV-VIS regions is associated with the interband transition and surface plasmon resonance of AuNPs, while the enhanced NIR absorption is due to the collective effect of interacting AuNPs in the close-packed network. Consequently, the AuNPs exhibits photodetection behavior in the wavelength range of 300-1500 nm. It is proposed that the inter-AuNP photoejection and delocalization of electron-hole pairs changes the carrier lifetime and transit dynamics in favor of photocarrier conduction, thus significantly facilitating photocurrent generation in the metallic AuNP close-pack. Moreover, due to the power-law conduction mechanism in AuNP networks, the quantum yield of AuNPs can be tuned from $10^{-6}$ to $10^{-1}$ photoelectron/photon by increasing the bias voltage from 0 to $5 \mathrm{~V}$. The AuNP quantum yield of $10^{-1}$ at $5 \mathrm{~V}$ is as high as that of commercial $\mathrm{Si}$ photodetectors at $0 \mathrm{~V}$, and this demonstrates the immediate applicability of AuNPs in photodetection. In view of the compatibility of AuNPs with wet-chemistry and inkjet printing processes at low temperatures, metallic AuNPs may provide a convenient alternative to semiconductor crystals in photodetection and perhaps photovoltaic applications. (C) 2010 American Institute of Physics. [doi:10.1063/1.3310367]
\end{abstract}

\section{INTRODUCTION}

Photocurrent generation and photodetection are usually based on semiconductor crystals such as $\mathrm{Si}, \mathrm{CdS}$, and $\mathrm{PbS}$, etc. ${ }^{1-4}$ One of the key factors for the high photocurrent $I_{p h}$ in semiconductors is the long lifetime $\tau$ of photoexcited electrons. The origin of the long lifetime is related to the energy band gap which determines the recombination rate of excited electron-hole $(e-h)$ pairs in semiconductors. In most cases, the conduction band photoelectrons do not recombine with holes by directly jumping through the band gap to the valence band. Instead, the recombination is mediated by imperfection states located in the band gap. ${ }^{1,2}$ Such an indirect recombination mechanism is responsible for the long lifetime (e.g., $\mu$ s-ms in Si) of semiconductors. Since photocurrent $I_{p h}$ is proportional to lifetime $\tau\left(I_{p h} \propto \tau\right),{ }^{1,2}$ large photocurrent is expected in semiconductor crystals.

In contrast, photocarrier lifetime in metals is ultrashort due to the lack of band gap. ${ }^{5}$ For instance, typical lifetime $\tau$ of 1-10 ps (for both electron-electron and electron-phonon interactions) is widely reported for metallic Au nanoparticles (NPs) ${ }^{5-8}$ In the case of metals, the photoexcited electrons can rapidly thermalize and decay via colliding with the large population of free conduction electrons near the Fermi surface. So the photoelectrons are too short-lived to participate in photocurrent conduction. This is why one does not expect

${ }^{a)}$ Electronic addresses: nnixxn@nus.edu.sg and phyweets@nus.edu.sg. photocurrent for metallic AuNPs, and why there are only two works on AuNP-based photoconductivity ${ }^{9,10}$ in the literature. Although we recently reported the photocurrent formation in AuNP films,${ }^{10}$ the most important issues, such as the mechanism and applicability of AuNP-based photocurrent, remain unaddressed. For example, the wavelength $\lambda$-dependence and quantum yield $Q Y$ of AuNP photocurrent are not known. In addition, there is no answer to the fundamental question on why photocurrent is possible in AuNPs even though the carrier lifetime is ultrashort.

In this work, we address the above issues by performing optical absorption and photocurrent measurements for closepacked AuNP network. We first show the photoabsorption of AuNPs throughout the UV-VIS (visible)-NIR (near infrared) region, and attribute the enhanced NIR absorption to the collective effect of interacting AuNPs. Consequently, AuNP photocurrent generation is observed in the wide 300-1500 $\mathrm{nm}$ wavelength range, and the strong voltage-dependence of $Q Y$ is discussed in terms of the power-law current-voltage (IV) characteristic of the AuNP network. Moreover, we also propose an inter-AuNP photoejection and $e-h$ pair delocalization model to explain the mechanism for photocurrent generation in metallic AuNPs.

\section{RESULTS AND DISCUSSION}

The AuNPs used in this work were synthesized according to previous works, ${ }^{10,11}$ and are of an average diameter of 

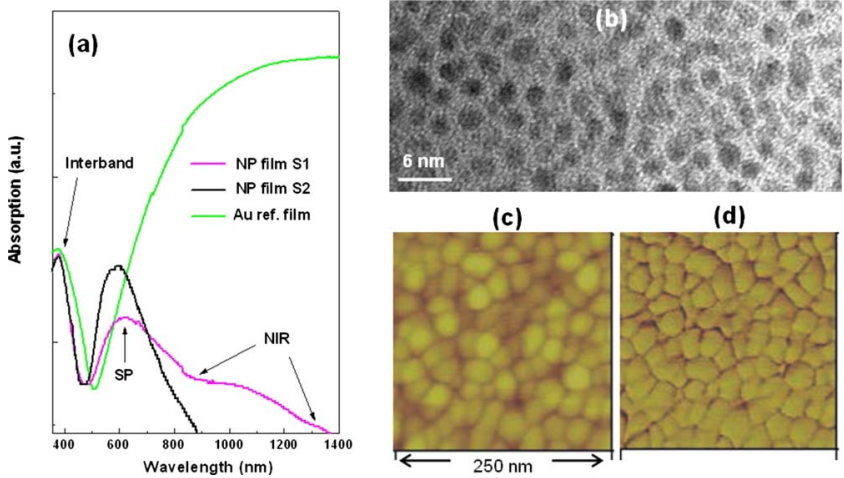

(c)

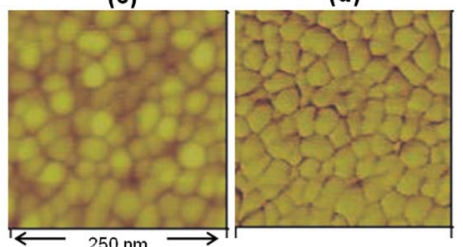

FIG. 1. (Color online) (a) Optical absorption spectra of close-packed and sparse-packed AuNP film S1 and S2, respectively. For comparison, the spectrum of a Au reference film consisting of very densely packed Au particles is also shown. The spectra are normalized to the interband peak at $380 \mathrm{~nm}$. (b) TEM image of the AuNPs with an average diameter of $3.3 \mathrm{~nm}$ used in this work. (c) and (d) are the AFM height and phase images of the Au reference film, respectively.

$3.3 \mathrm{~nm}$ as shown in the TEM (transmission electron microscope) image in Fig. 1(b) [see Ref. 12, Fig. i(a) for experimental details]. The AuNPs were spin-coated onto transparent indium tin oxide (ITO) glass slides to form thin films for optical absorption measurements. Two AuNP films, film S1 consisting of close-packed AuNPs and film S2 composed of sparse-packed AuNPs, were prepared for comparison of their absorption behaviors [see Ref. 12, Fig. i(b)]. Figure 1(a) displays the optical absorption spectra collected for AuNP films S1 and S2, respectively (see Ref. 12, Fig. ii for experimental details). In the UV region in Fig. 1(a), a prominent peak at $380 \mathrm{~nm}$ is observed for film S1, and the peak corresponds to the $5 d \rightarrow 6 s p$ interband transition ${ }^{13-15}$ of AuNPs. In the VIS region, the well-known surface plasmon (SP) peak ${ }^{16-18}$ of AuNPs is centered at $620 \mathrm{~nm}$. Most importantly, an absorption shoulder in the $850-1400 \mathrm{~nm}$ NIR region is observed for film S1. The NIR absorption shoulder was further confirmed by measuring the absorption spectrum of AuNP film S2 which consists of sparse-packed AuNPs. In the case of film $\mathrm{S} 2$, the interband peak is located at the same position as that of film S1, but the SP peak is blue-shifted to $589 \mathrm{~nm}$. This absorption behavior is quite similar to that observed for isolated individual AuNPs. ${ }^{16-18}$ Since the spectra are normalized to the interband peak, it is clear that the SP peak of film $\mathrm{S} 2$ is narrower than that of film S1. In addition, the SP absorption drops to baseline level near $900 \mathrm{~nm}$, thus confirming the existence of the NIR absorption identified for film S1. Since the major difference between the two films is the close- and sparse-packing of AuNPs, we thus attribute the NIR shoulder to the collective interaction of close-packed AuNPs in film S1. In the literature, there are reports on enhanced NIR absorption by metallic nanostructures, and the aggregation of NPS and the inter-NP interaction are proposed to be the origins. ${ }^{19-21}$ Here we believe the collective effect of interacting AuNPs in the close-packed three-dimensional (3D) NP network is responsible for the enhanced NIR absorption observed in Fig. 1(a). To confirm our hypothesis, we used electron-beam-deposition method to prepare a Au reference film which consists of very densely packed Au particles as shown in the atomic force microscope (AFM) im-

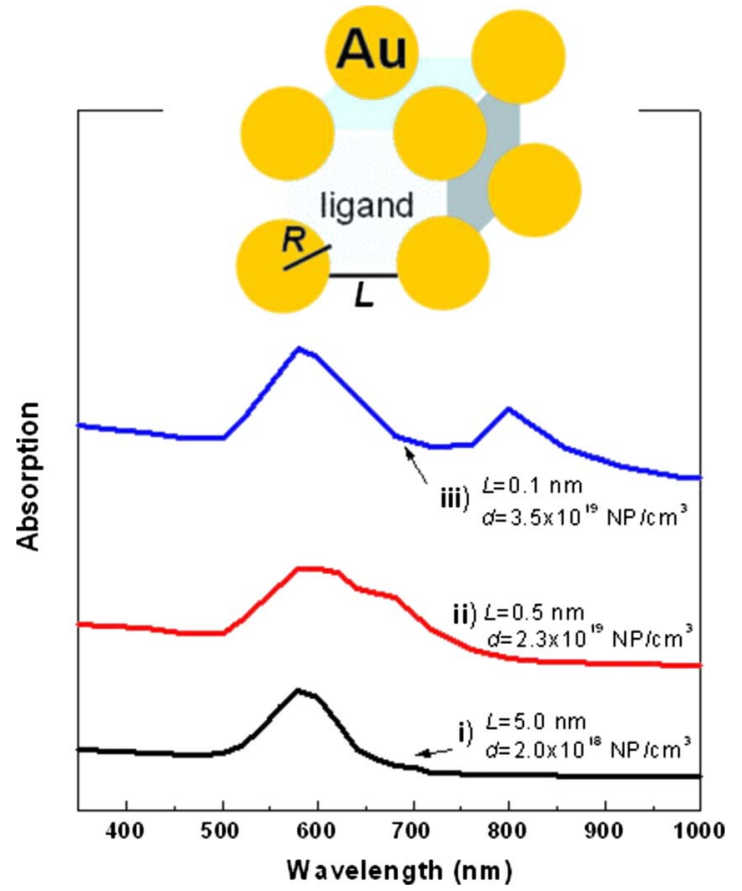

FIG. 2. (Color online) Evolution of simulated optical absorption of AuNP ensembles with different packing density $d$. The inset shows the simple cubic packing of AuNPs assumed in the simulation: a constant NP radius of $R=1.5 \mathrm{~nm}$ is kept, while the inter-NP spacing $L$ is varied to mimic different NP packing density $d$. The intensity of the spectra has been adjusted for easy comparison of the peak position and shape.

ages in Figs. 1(c) and 1(d) (see SI- $i-c$ for experimental). The average diameter of the Au particles is $\sim 31 \mathrm{~nm}$, and the high packing density of the reference film is clearly viewed in the AFM phase image [see Fig. 2(c)] which better resolves the boundary of particles than the height image does. For such a densely packed metallic Au particle network, the enhanced NIR absorption is so strong that it surpasses the SP absorption, thus making the SP peak undistinguishable from the broad NIR absorption peak [see Fig. 1(a)]. Note that the size of the electron-beam-deposited Au particles is much larger than that of the synthesized AuNPs [see Figs. 1(b) and 1(c)], so the difference in absorption shown in Fig. 1(a) may also be caused by the change in particle size. However, our theoretical simulation discussed in Fig. 2 below indicates that the optical absorption is dominated by the interaction of Au particles.

The observation in Fig. 1 highlights the critical role of inter-AuNP coupling in the determination of the optical behaviors of a AuNP ensemble. For a $N$-particle system, its optical response is simply the $N$-fold of the individuals only when the particles are well-separated. In most cases, the interparticle interaction contributes significantly to the optical property, and the overall absorption is a combination of both single-particle and collective effects of the NP ensemble. The enhanced NIR absorption in Fig. 1(a) is usually not observable in a homogenous bulk Au film because the intraband transition is restricted by: (i) the momentum mismatching between incident photons and conduction band electrons and (ii) the dipole forbiddance of conduction band electrons in terms of symmetry rules. ${ }^{22-24}$ In the case of our AuNP closepack, the collective effect associated with NP interaction 
(a)

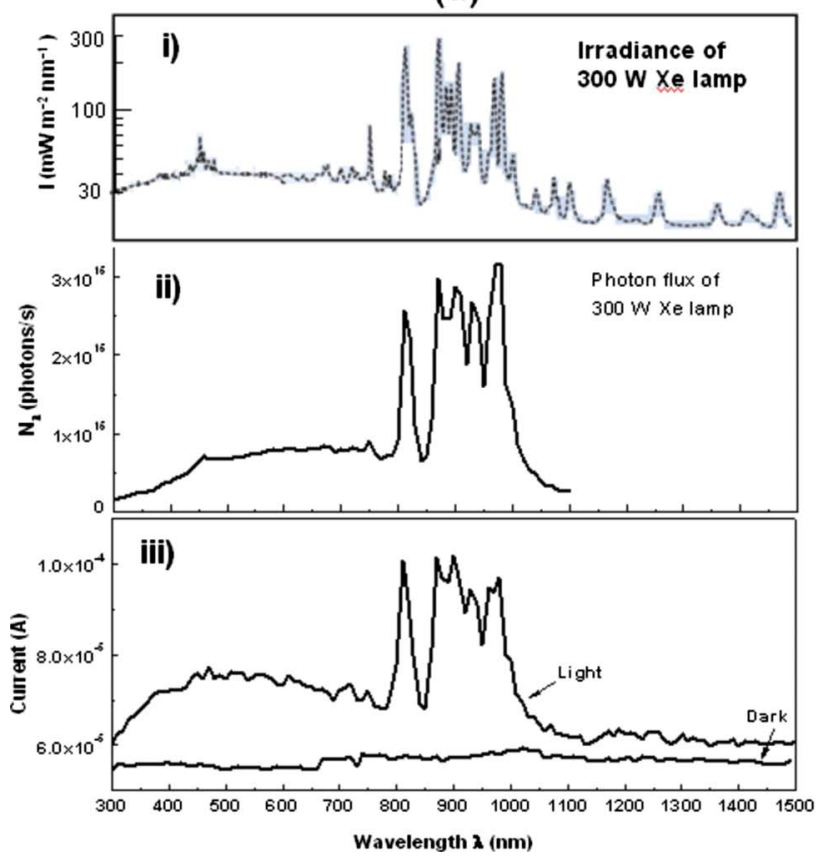

(b)

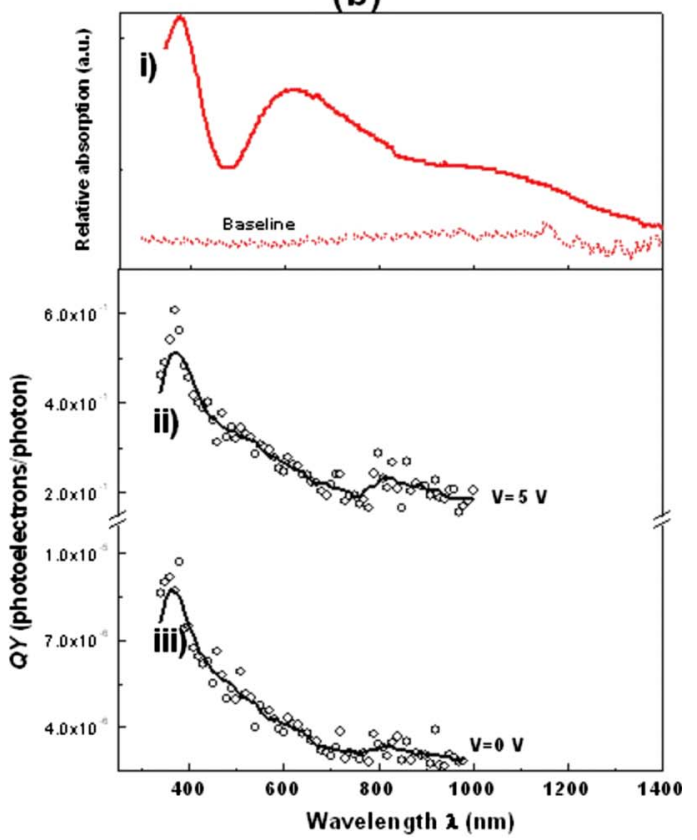

FIG. 3. (Color online) (a): (i) irradiance spectrum (at $0.5 \mathrm{~m}$ ) of the $300 \mathrm{~W}$ Xe lamp used in the experiments. The spectrum is reproduced from Ref. 29; (ii) photon flux of the Xe lamp determined using a calibrated commercial Si photodetector; and (iii) photocurrent and dark current recorded for AuNPs. (b): (i) Optical absorption spectrum of the close-packed AuNP network; (ii) and (iii) are the quantum yield spectra of AuNPs obtained under 5 and 0 V bias voltage, respectively.

such as multipole coupling reduces the two restrictions, thus enabling the intraband transition in the NIR region. This enhancement can be simulated by assuming a simple cubic packing of AuNPs in the alkyl ligand medium [see Ref. 12, Fig. iii (Refs. 25-27)] as shown in Fig. 2. The inset of Fig. 2 displays the cubic packing of AuNPs in which a constant NP radius of $R=1.5 \mathrm{~nm}$ is assumed, and different inter-NP separation $L$ is considered to represent different NP packing density $d$. For sparse-packed NPs, e.g., $L=5.0 \mathrm{~nm}$ or $d=2.0$ $\times 10^{18} \mathrm{NP} / \mathrm{cm}^{3}$, the absorption [see spectrum-i in Fig. 2] is dominated by a SP peak at $580 \mathrm{~nm}$, which is characteristic of the absorption reported for individual AuNPs. ${ }^{16-18}$ In the case of medium-packed NPs, the SP peak broadens obviously, and an absorption shoulder develops at $670 \mathrm{~nm}$ as shown in spectrum-ii calculated for $L=0.5 \mathrm{~nm}$ in Fig. 2 . This broadening effect is due to the onset of inter-NP coupling when NPs are brought closer in the medium-packed AuNP ensemble. For close-packed NPs with $L \leq 0.1 \mathrm{~nm}$, a second absorption peak is observed at $800 \mathrm{~nm}$ in the NIR region [see spectrum-iii in Fig. 2]. Therefore, the evolution of absorption as a function of AuNP separation or packing density confirms that the NIR absorption experimentally observed in Fig. 1(a) is induced by inter-NP coupling in the close-packed NP network. Note that the NIR absorption simulated in Fig. 2 is obtained by considering the interaction of AuNPs only. If the disorder and size distribution of $\mathrm{NPs}^{22-24}$ are further considered, the simulated absorption spectra would be closer in fine structure to the experimental spectra. Nonetheless, the above simplified simulation captures the key effect of NP interaction on NIR absorption, and is qualitatively in agreement with our experimental observations.
On the basis of photoabsorption presented in Figs. 1 and 2, we further demonstrate the AuNP-based photocurrent generation and photodetection in the UV-VIS-NIR region. The photocurrent measurements were conducted using the light source (i.e., $300 \mathrm{~W}$ Xe lamp) and Si photodetector of the Oriel system purchased from Newport Corporation. ${ }^{28,29}$ AuNP films consisting of close-packed AuNPs similar to film S1 were spin-coated on intrinsic p-type Si substrate, and ITO glass was used as top electrode to form ITO/AuNPs/p-Si device configuration (see Ref. 12, Fig. iv for experimental details). Figure 3(a)-iii shows the evolution of photocurrent observed for the AuNP film in the wavelength range of $\lambda$ $=300-1500 \mathrm{~nm}$. A comparison between the light current and dark current (obtained without light irradiance) clearly indicates the generation of photocurrent by the AuNP network. Figure 3(a)-i displays the typical irradiance of the 300 W Xe lamp in the range of 300-1500 nm (reproduced from Ref. 29). The close similarity between the light irradiance and the AuNP photocurrent suggests that the photocurrent is strongly affected by light intensity. To obtain the photocurrent quantum yield $Q Y$, i.e., the number of photoelectrons generated per photon of a specific wavelength $\lambda$ (or energy $h v$ ), we used the commercial Si photodetector ${ }^{28}$ to measure the $\lambda$-dependent photon flux of the Xe lamp. Figure 3(a)-ii displays the corresponding number $N_{\lambda}$ of photons generated by the Xe lamp which was determined on the basis of the photocurrent and photoresponsivity of the calibrated Si detector shown in Fig. S1 (see Ref. 12, Fig. v(a) for details). The values of $N_{\lambda}$ is shown only in the 300-1100 $\mathrm{nm}$ range in Fig. 3(a)-ii because the detection range of the Si detector is up to $1100 \mathrm{~nm}$. The number $N_{p h-e}$ of photoelectrons generated in the AuNP network by the photon flux can be esti- 
mated using the photocurrent data shown in Fig. 3(a)-iii. The $Q Y$ of AuNPs is then calculated by taking the ratio $Q Y$ $=N_{p h-e} / N_{\lambda}$ for each wavelength $\lambda$ [see Ref. 12, Fig. v(b)]. Figure 3(b)-ii and iii displays the $Q Y$ thus obtained under a bias voltage of $V=0$ and $5 \mathrm{~V}$, respectively. At $V=5 \mathrm{~V}$, the $Q Y$ is in the order of $10^{-1}$ photoelectrons/photon, and the highest $Q Y$ is observed in the UV region. Then the $Q Y$ gradually decreases throughout the VIS region. Interestingly, a $Q Y$ peak appears in the NIR region $(850-1000 \mathrm{~nm})$. Similar $Q Y$ results are also observed for $V=0 \mathrm{~V}$, although at $0 \mathrm{~V}$, the $Q Y$ is as low as $10^{-6}$ [see Fig. 3(b)-iii].

To interpret the $\lambda$-dependence of AuNP $Q Y$, we show the optical absorption spectrum of AuNP film S1 [already presented in Fig. 1(a)] as Fig. 3(b)-i. $Q Y$ is usually determined by the absorption coefficient $\alpha$ and the photon energy $h v$, e.g., $Q Y \propto \alpha h v .{ }^{1,2}$ The high $Q Y$ in the UV region in Fig. 3(b)-ii and iii is a result of the enhanced interband absorption [or higher $\alpha$ value, see the absorption peak at $380 \mathrm{~nm}$ in Fig. $3(\mathrm{~b})-\mathrm{i}]$ and the higher energy $h v$ of the UV photons. Although the SP peak around $620 \mathrm{~nm}$ is quite obvious in the absorption spectrum in Fig. 3(b)-i, there is no corresponding peak in the $Q Y$ spectra in Fig. 3(b)-ii and iii. This can be attributed to the decrease in $h v$ as $\lambda$ increases, and the dominance of $h v$ over $\alpha$ in the determination of $Q Y$. The $Q Y$ peak in the 750-1000 $\mathrm{nm}$ region coincides with the enhanced NIR absorption as discussed in Figs. 1 and 2. In this region, the intraband transition is greatly enhanced due to the collective interaction of close-packed AuNPs. Since there are many free electrons in the conduction band of metallic AuNPs, the enhanced absorption generates considerable photoelectrons, thus leading to the NIR photocurrent peak in the $Q Y$ spectrum. Because of the detection limit of the Si detector, we were not able to collect the accurate Xe lamp photon flux above $1000 \mathrm{~nm}$, so the $Q Y$ spectrum in the $1000-1400 \mathrm{~nm}$ region was not obtained in this work. However, the broad NIR absorption shoulder (up to $1400 \mathrm{~nm}$ ) in Fig. 3(b)-i and the appreciable photocurrent observed in the 1000-1400 nm range in Fig. 3(a)-iii, are good indications of photoabsorption and photocurrent generation in this region.

We further propose an inter-AuNP photoejection and $e-h$ pair delocalization model to qualitatively clarify the concern about AuNP-based photocurrent arising from the ultrashort carrier lifetime $\tau$ of $1-10$ ps (Refs. 5-8) in metallic AuNPs. In this model shown in Fig. 4(a), photoelectrons excited from AuNP1 are not confined in NP1, instead, they can be directly photoejected into AuNP2 through the lowest unoccupied molecular orbital of the ligand. This is possible because the enhanced interband and intraband excitations in AuNPs (see Figs. 1 and 2) are able to generate photoelectrons whose energy is high enough to overcome the barrier $\phi$. Such photoejection would be more prominent under our experimental conditions of continuous photon irradiance. When an electron is ejected from AuNP1 to AuNP2, the hole $(h)$ remains in AuNP1 while the electron $(e)$ is delocalized to AuNP2, thus making the recombination of the $e$ - $h$ pair difficult due to their different locations. The separated $e-h$ pair would thus remain free for a longer time, and this is equivalent to an increase in carrier lifetime $\tau$. In addition, under enhanced photon excitation, the photoelectrons can be ejected not only (a)
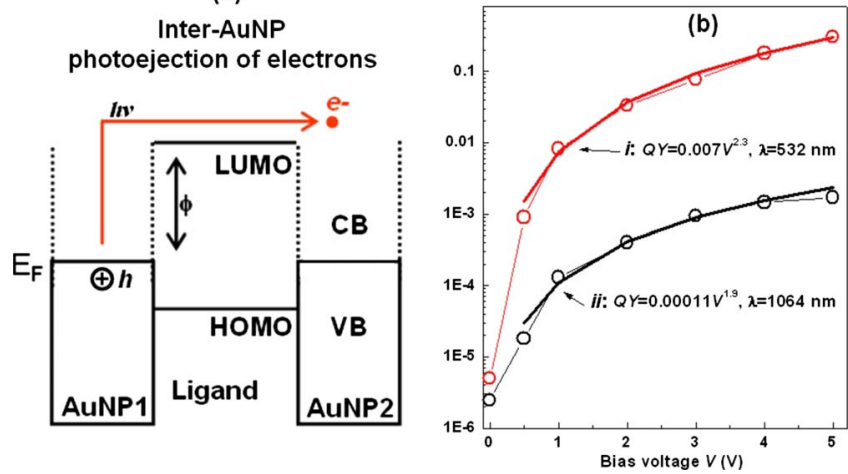

FIG. 4. (Color online) (a) Proposed inter-AuNP photoejection and photoelectron delocalization model. (b) Bias-dependence of $Q Y$ observed for $\lambda$ $=532$ and $1064 \mathrm{~nm}$, respectively. The empty circles are the experimental data, while the solid lines are fits to the power-law.

to the nearest neighbor NPs, but also to the distant NPs in the network. This effect is associated with a reduction in the transit time $t_{0}$ needed for the photoelectrons to drift through the thickness $d_{0}$ of the AuNP film. Since photocurrent can also be expressed as $I_{p h} \propto\left(\tau / t_{0}\right),{ }^{1,2}$ it is clear that the increased $\tau$ and decreased $t_{0}$ can both lead to an increase in $I_{p h}$. Usually the delocalization of photoelectron is achieved by electron hopping shown in Fig. 4(a), and its typical time scale per hopping event is in the order of picosecond-nanosecond. ${ }^{30}$ In our case of $d_{0}=100 \mathrm{~nm}$ (corresponding to 25-hopping events), a conservative estimation of $\tau / t_{0}$ up to $10^{-1}$ can be obtained. So even though the absolute lifetime $\tau$ is very short, the relative ratio $\tau / t_{0}$ can be made large enough by photoejection. Therefore, the opposite change in photoelectron recombination and transport dynamics associated with the delocalization of $e-h$ pairs allows for photocurrent generation in the AuNP network.

Plot-i in Fig. 4(b) displays the variation in $Q Y$ as a function of bias voltage $V$ observed for a single wavelength $\lambda$ $=532 \mathrm{~nm}$ of the Xe lamp. The $Q Y$ is in the order of $\sim 10^{-6}$ at zero bias, but it jumps to $\sim 10^{-1}$ when the bias is increased to $5 \mathrm{~V}$. Such a strong bias-dependence of $Q Y$ is another important feature of AuNP-based photocurrent, and it indicates the $Q Y$ can be tuned to a high yield comparable to that of the commercial Si photodetector $\left(\sim 10^{-1}\right.$ at zero bias as derived from the photoresponsivity shown in Fig. S1 and Ref. 28) by applying a moderate bias of $5 \mathrm{~V}$. We propose that the biasdependence of $Q Y$ is a unique character of the AuNP system. For such a system, the $(I V)$ characteristic is usually governed by a power-law ${ }^{31-37} I=B\left(V-V_{T}\right)^{\zeta}$, where $B$ is a constant, $V_{T}$ is the threshold voltage for the onset of charge conduction, and $\zeta$ is the nonlinear exponent whose value depends on the dimensionality of the AuNP assembly. This power-law is an outcome of inter-AuNP electron hopping/tunneling, and as the dimensionality of the AuNP assembly increases, the number of nearest neighbors of a AuNP also increases, thus creating more tunneling paths and generating larger current in the network. The theoretical value of $\zeta$ is 1.0 and $\frac{5}{3}$ for onedimensional and two-dimensional (2D) NP arrays, respectively. ${ }^{31}$ The threshold voltage $V_{T}$ arises from the Coulomb blockade energy of a NP, and is temperature- and dimensionality-dependent. ${ }^{32-34}$ In 2D/3D AuNP arrays at 
room temperature, $V_{T}$ becomes negligibly small due to the formation of percolating paths in the close-packed assembly. ${ }^{36}$ The nonlinear plot-i in Fig. 4(b) suggests that photocurrent conduction is also governed by the power-law of the AuNP network. Indeed, plot-i can be fitted to the form $Q Y=0.007 V^{2.3}$ [see the solid line of plot-i in Fig. 4(b)]. To confirm the results obtained using the Xe lamp, we also used two individual laser diodes which generate photon flux of a single wavelength of 532 and $1064 \mathrm{~nm}$, respectively (see Ref. 12, Fig. vi). The $Y Q \sim V$ plot thus obtained using the $532 \mathrm{~nm}$ green laser diode [see Fig. S4(b)] is very similar to the one collected using the Xe lamp, and the plot corresponding to the $1064 \mathrm{~nm}$ NIR laser diode is shown as plot-ii in Fig. 4(b). This plot can also be fitted to the power-law in the form $Q Y=0.00011 V^{1.9}$. The fitting of plot-i and ii corresponds to $V_{T} \approx 0 \mathrm{~V}$ and $\zeta=1.9-2.3$, both of which are in good agreement with our early observation ${ }^{10}$ and results reported by others for similar 2D/3D AuNP assemblies. ${ }^{31-36}$ The different prefactor of 0.007 for plot-i and 0.00011 for plot-ii reflects the different photocurrent generation efficiency of the 532 and $1064 \mathrm{~nm}$ photons, respectively. The above $Q Y \sim V$ power-law associated with the incoherent charge hopping in the heterogeneous AuNP/ligand system is fundamentally different from the linear relation commonly observed for semiconductors. ${ }^{1,2}$ In the latter case of homogeneous band gap materials, coherent charge transport through the conduction band is predominant. Therefore, the incoherent powerlaw charge conduction mechanism in ligated AuNP ensembles is responsible for the rapid increase in $Q Y$ by several orders within a small bias range of $0-5 \mathrm{~V}$ observed in Fig. 4(b).

It is instructive to briefly summarize the new behaviors of AuNP network in terms of its photoabsorption and photoconductivity. The results in Fig. 1 demonstrate the photoabsorption of AuNPs in the broad UV-VIS-NIR region. The UV absorption is related to the enhanced interband transition in AuNPs, while the VIS absorption is associated with the SP resonance typical of noble metals. In particular, the NIR absorption, which is not observed for individual NPs, is induced by the collective interaction of close-packed AuNPs as discussed in Figs. 1 and 2. Such enhanced absorption allows for photocurrent generation and photodetection in the wide wavelength range of 300-1100 $\mathrm{nm}$. Due to the photoejection and $e-h$ pair delocalization effect, the carrier lifetime and transit dynamics are changed in favor of photoelectron conduction in the AuNP network [see Fig. 4(a)]. Although the $Q Y$ of AuNPs is low $\left(10^{-6}\right)$ at zero bias, the power-law biasdependence of $Q Y$ enables the significant increase in $Q Y$ to $10^{-1}$ at a bias of $5 \mathrm{~V}$ [see Fig. 4(b)], and the value is in the same order as the $Q Y$ of commercial Si photodetector. This is quite remarkable as it suggests the immediate applicability of AuNPs in photodetection. Indeed, the evolution of AuNP photocurrent in response to Xe lamp light irradiance in the broad $300-1500 \mathrm{~nm}$ range is a good indication of its photodetection performance [see Fig. 3(a)].

In conclusion, we observed enhanced optical absorption in the UV-VIS-NIR region that is associated with the size and collective effects of metallic AuNPs. Consequently, the close-packed AuNP network is capable of photodetection in the wavelength range of 300-1500 nm. Photocurrent generation in the AuNP network is facilitated by inter-AuNP photoejection and $e-h$ pair delocalization. Most importantly, the quantum yield of the AuNP-based photodetection can be tuned to $10^{-1}$ photoelectrons/photon by the application of a small bias voltage of $5 \mathrm{~V}$. This quantum yield is comparable to that of commercial Si photodetectors under zero bias, and indicates the immediate possibility of AuNPs in photodetection. Being solution processable and inkjet printable, ${ }^{11,38-40}$ metallic AuNPs may serve as a flexible alternative to conventional semiconductor crystal-based photodetection.

\section{ACKNOWLEDGMENTS}

We greatly thank Mr. S. Sivaramakrishnan, Mr. B. T. Anto, and Dr. P. K.-H. Ho of the Organic NanoDevices Laboratory (ONDL, Physics Department at NUS) for kindly providing the various Au NP films and performing TEM characterization of the Au NPs. Mr. Kian Keat Lee is gratefully acknowledged for the electron-beam deposition of $\mathrm{Au}$ films. This work is supported by NUS Nanoscience and Nanotechnology Initiative (NUSNNI), National University of Singapore.

${ }^{1}$ R. H. Bube, Photoconductivity of Solids (Wiley, New York 1960).

${ }^{2}$ R. H. Bube, Photoelectronic Properties of Semiconductors (Cambridge University Press, Cambridge, London, 1992).

${ }^{3}$ S. McDonald, G. Konstantatos, S. Zhang, P. W. Cyr, E. J. D. Klem, L. Levina, and E. H. Sargent, Nature Mater. 4, 138 (2005).

${ }^{4}$ E. H. Sargent, Adv. Mater. (Weinheim, Ger.) 17, 515 (2005).

${ }^{5}$ J. Z. Zhang, Acc. Chem. Res. 30, 423 (1997).

${ }^{6}$ S. Link and M. A. El-Sayed, Annu. Rev. Phys. Chem. 54, 331 (2003).

${ }^{7}$ C. Voisin, D. Christofilos, N. D. Fatti, F. Vallee, B. Prevel, E. Cottancin, J. Lerme, M. Pellarin, and M. Broyer, Phys. Rev. Lett. 85, 2200 (2000).

${ }^{8}$ O. P. Varnavski, T. Goodson III, M. B. Mohamed, and M. A. El-Sayed, Phys. Rev. B 72, 235405 (2005)

${ }^{9}$ N. Fishelson, I. Shkrob, O. Lev, J. Gun, and A. D. Modestov, Langmuir 17, 403 (2001)

${ }^{10}$ X. N. Xie, Y. Xie, X. Gao, C. H. Sow, and A. T. S. Wee, Adv. Mater. (Weinheim, Ger.) 21, 3016 (2009)

${ }^{11}$ S. Sivaramakrishnan, P.-J. Chia, Y.-C. Yeo, L.-L. Chua, and P. K.-H. Ho, Nature Mater. 6, 149 (2007).

${ }^{12}$ See supplementary material at http://dx.doi.org/10.1063/1.3310367 that contains the details for (i) the synthesis of AuNPs and AuNP film deposition; (ii) the optical absorption measurements and simulation of optical absorption; (iii) photocurrent measurements and the determination of AuNP photocurrent quantum yield.

${ }^{13}$ B. Balamurugan and T. Maruyama, Appl. Phys. Lett. 87, 143105 (2005).

${ }^{14}$ X. N. Xie, X. Gao, D. Qi, Y. Xie, L. Shen, S.-W. Yang, C. H. Sow, and A. T. S. Wee, ACS Nano 3, 2722 (2009).

${ }^{15}$ M. Haridas, S. Srivastava, and J. K. Basu, Eur. Phys. J. D 49, 93 (2008).

${ }^{16}$ S. Link and M. A. El-Sayed, Int. Rev. Phys. Chem. 19, 409 (2000).

${ }^{17}$ H. J. Shin, I.-W. Hwang, Y.-N. Hwang, D. Kim, S. H. Han, J.-S. Lee, and G. Cho, J. Phys. Chem. B 107, 4699 (2003).

${ }^{18}$ S. Link and M. A. El-Sayed, J. Phys. Chem. B 103, 4212 (1999).

${ }^{19}$ E. Hutter and J. H. Fendler, Adv. Mater. (Weinheim, Ger.) 16, 1685 (2004).

${ }^{20}$ T. J. Norman, C. D. Grant, D. Magana, J. Z. Zhang, J. Liu, D. Cao, F. Bridges, and A. V. Buuren, J. Phys. Chem. B 106, 7005 (2002).

${ }^{21}$ A. N. Shipway, M. Lahav, R. Gabai, and I. Willner, Langmuir 16, 8789 (2000).

${ }^{22}$ U. Kreibig and L. Genzel, Surf. Sci. 156, 678 (1985).

${ }^{23}$ M. R. Beversluis, A. Bouhelier, and L. Novotny, Phys. Rev. B 68, 115433 (2003).

${ }^{24}$ J. R. Zurita-Sánchez and L. Novotny, J. Opt. Soc. Am. B 19, 1355 (2002).

${ }^{25}$ B. T. Draine and P. J. Flatau, J. Opt. Soc. Am. A Opt. Image Sci. Vis 11, 1491 (1994).

${ }^{26}$ B. T. Draine and P. J. Flatau, e-print arXiv:0809.0337 (2008).

${ }^{27}$ J. J. Ritsko, J. Chem. Phys. 70, 5343 (1979). 
${ }^{28} \mathrm{http}: / /$ www.newport.com/Oriel-Silicon-Detectors/378913/1033/ catalog.aspx

${ }^{29} \mathrm{http} / / /$ www.newport.com/file_store/Supporting_Documents/

Tech_Ref_Spectral_Irradiance25.pdf. (See Fig. 6 on page 24 for the irradiance spectrum of the $300 \mathrm{~W}$ Xe lamp. See page 3 for the calculation of the number of photons in a photon flux with wavelength $\lambda$ and power W.) ${ }^{30}$ C. Wasshuber, Computational Single-Electronics (Springer, New York, 2001).

${ }^{31}$ A. A. Middleton and N. S. Wingreen, Phys. Rev. Lett. 71, 3198 (1993).

${ }^{32}$ R. W. Rendell, M. G. Ancona, W. Kruppa, E. E. Foos, A. W. Snow, D. Park, and J. B. Boos, IEEE Trans. Nanotechnol. 2, 75 (2003).

${ }^{33}$ R. Parthasarathy, X.-M. Lin, K. Elteto, T. F. Rosenbaum, and H. M. Jae- ger, Phys. Rev. Lett. 92, 076801 (2004).

${ }^{34}$ R. Parthasarathy, X.-M. Lin, and H. M. Jaeger, Phys. Rev. Lett. 87, 186807 (2001).

${ }^{35}$ U. Simon, Adv. Mater. (Weinheim, Ger.) 10, 1487 (1998).

${ }^{36}$ A. Taleb, F. Silly, A. O. Gusev, F. Charra, and M.-P. Pileni, Adv. Mater. (Weinheim, Ger.) 12, 633 (2000).

${ }^{37}$ Z. Huang, J. E. Carey, M. Liu, X. Guo, E. Mazur, and J. C. Campbell, Appl. Phys. Lett. 89, 033506 (2006).

${ }^{38}$ M.-C. Daniel and D. Astruc, Chem. Rev. (Washington, D.C.) 104, 293 (2004).

${ }^{39}$ P. Moriarty, Rep. Prog. Phys. 64, 297 (2001).

${ }^{40}$ F. Baletto and R. Ferrando, Rev. Mod. Phys. 77, 371 (2005). 